\title{
Study on Synthesis Method of Multipoint Seismic Waves for Buried Oil and Gas Pipeline in Shaking Table Tests
}

\author{
Jian-Bo Dai $\mathbb{D}^{\mathbb{D}}$, Gui-Di Zhang ${ }^{\mathbb{D}}$, Cheng-Tao Hu $\mathbb{D}^{\text {, }}$, and Kai-Kai Cheng \\ Collage of Mechanical Engineering, Xi'an Shiyou University, Xi'an 710065, China \\ Correspondence should be addressed to Jian-Bo Dai; 184298843@qq.com
}

Received 16 May 2021; Revised 5 July 2021; Accepted 27 July 2021; Published 2 August 2021

Academic Editor: Angelo Marcelo Tusset

Copyright ( $\odot 2021$ Jian-Bo Dai et al. This is an open access article distributed under the Creative Commons Attribution License, which permits unrestricted use, distribution, and reproduction in any medium, provided the original work is properly cited.

\begin{abstract}
The buried oil and gas pipeline is a linear structure with infinite length. In the shaking table test of its seismic response, it is necessary to input the spatially related multipoint seismic wave considering the propagation characteristics of ground motion. The multipoint seismic excitation shaking table tests and loading scheme of buried oil and gas pipelines are designed and formulated. The synthesis method of spatial correlation multipoint seismic wave for the buried oil and gas pipeline test is proposed in this study. The values of relevant parameters are analyzed, and corresponding program is compiled by MATLAB. The results show that the developed multipoint excitation shaking table seismic wave input scheme is reasonable. At the same time, the synthesized multipoint seismic wave based on the actual seismic record and artificial random simulation seismic wave can meet the test requirements, which suggests the testing effect is good.
\end{abstract}

\section{Introduction}

Buried oil and gas pipeline is called lifeline project of oil and gas field. Investigation on serious earthquake disasters shows that not only is the buried pipeline directly damaged in the earthquake, but also it can produce seriously secondary disasters such as fire and explosion and environmental pollution. The buried oil and gas pipeline is an infinite structure. Before seismic wave acts on the pipeline and its surrounding soil, it needs to pass through complex geological conditions. Affected by the traveling wave effect, local site effect, and partial coherence effect, the velocity, timeconsuming, and amplitude of the seismic wave propagating to each point of the pipeline are different. Therefore, it is necessary to fully realize the randomness and spatial variability of ground motion and the correlation of ground motion at each point in the seismic field and use spatial multipoint seismic excitation for seismic analysis of longdistance pipelines.

It is an effective method to analyze the seismic response of long linear structure by using a multipoint seismic excitation test with a multiseismic simulation shaking table array. However, some research studies have been carried out, in which the seismic wave with traveling wave effect is input to the shaking table test. Han et al. [1] carried out a shaking table test of buried pipeline under multipoint nonuniform excitation, but only the influence of traveling effect during the ground motion propagation was considered in seismic wave input, and the time delay of each shaking table was $1 \mathrm{~s}$. $\mathrm{Du}$ et al. [2] investigated the nonlinear soil response under nonuniform seismic excitation in the shaking table test. Aspasia et al. [3] studied the response of pipelines under random seismic action and analyzed the effects of factors such as the incidence angle of seismic waves at different locations in the longitudinal direction, the arrival time of fluctuations, and different fluctuation characteristics.

The above shaking table test only considers the traveling wave effect of seismic wave propagation, while the test using different array records of actual seismic wave or multipoint excitation seismic wave with spatial synthesis correlation is more consistent with the actual situation of seismic wave propagation. Therefore, it is necessary to synthesize multipoint nonstationary space seismic waves for experiment and numerical simulation analysis. Okawa [4] proposed two types of envelope functions, one in which the motion of one or more expected ground envelopes occurs at a certain time, 
and the other in which the envelope function is generated based on earthquake magnitude, distance, and site conditions. Hai et al. [5] conducted the shaking table test of pipeline-soil seismic response under the nonuniform excitation. During the trial, EL-Centro wave was selected as the original seismic wave to obtain the cross power spectrum and the spectral representation method was used to generate the time history of multipoint stable ground motion, which was multiplied by the envelope function to form a nonstationary artificial ground motion random process from which two related seismic waves were synthesized, to be used as the input wave of each shaking table. Kerry [6] has generated a record of seismic waves using the reflection method. This method provides a convenient framework, and the principle is to use the method of dispersion diagram and mode summation. The seismic waves can be generated in a comprehensive manner considering that seismic waves travel through complex media with different propagation velocities and superposition of seismic wave components at different frequencies during propagation. Deodatis [7] proposed a simulation algorithm based on the spectral representation to generate sample functions that employ the mutual power spectral density function to generate nonsmooth random seismic waves.

Aiming at establishing the scale model of buried oil and gas pipeline and its surrounding soil, a bidirectional laminated shear continuum model box is designed. The double array shaking table is used for seismic excitation to study the seismic response of buried oil and gas pipelines under uniform and multipoint seismic excitation. Based on the actual seismic records, two multipoint seismic waves and a random artificial multipoint seismic wave are synthesized randomly.

\section{Shaking Table Tests for Buried Oil and Gas Pipeline}

The test is carried out on the horizontal two-way seismic simulation vibration array system of Hunan Key Laboratory of structural wind resistance and vibration control. The shaking table can realize two-way four degrees of freedom loading. The size of single table is $1.000 \mathrm{~m} \times 1.000 \mathrm{~m}$, maximum load is $50 \mathrm{kN}$, the working frequency band is $0.1-50 \mathrm{~Hz}$, and maximum horizontal displacement is $\pm 0.075 \mathrm{~m}$. In order to study the acceleration response of the pipeline and soil and the strain response of the pipeline, a total of 24 resistive strain gauges and 5 three-way acceleration sensors were installed on the pipeline, 1 acceleration sensor on each of the left and right vibration tables, and 9 acceleration sensors in the soil. A dynamic data acquisition instrument was used to collect and analyze the data, and the accuracy level of the resistive strain gauges and acceleration sensors was A grade to simulate as realistically as possible the vibration response of the oil and gas long-distance pipeline under ground shaking and to reduce the errors in the test. The multipoint seismic excitation vibration array test is shown in Figure 1. During the test, the shaker needs to input the acceleration value to calculate the power spectrum and response spectrum of the response according to the model,

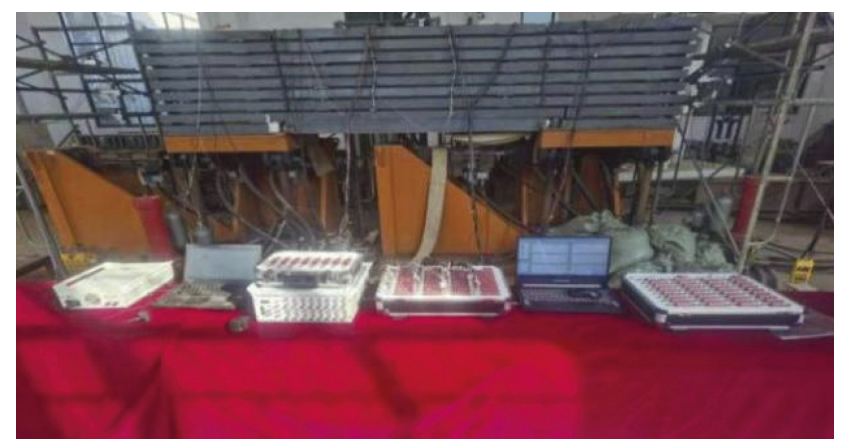

Figure 1: Multipoint seismic excitation vibration array test.

and each test only needs to set the loading acceleration value to get the corresponding multipoint correlation seismic excitation time curve through the model calculation.

According to the similarity ratio design, a model of the scaled-down pipe and the surrounding soil was made by $1 / 10$, and a bidirectional laminated shear continuum model box was designed and made. The soil box is divided into three sections, the left and right boxes are placed on the shaking table, the bottom of the middle-box can slide on the shaking table at both ends, and each section is connected by an articulated telescopic device, so that the box can undergo bidirectional noncoherent horizontal motion during multipoint seismic wave input in the table and also simulate the laminar shear effect between soil. The pipe model was $0.140 \mathrm{~m} \times 0.003 \mathrm{~m}$ in cross section and $3.500 \mathrm{~m}$ in length. The interior of the model pipe was pressurized to $8 \times 10^{6} \mathrm{~Pa}$ before testing to simulate the pressure generated by the internal media on the pipe wall during oil and gas transportation.

During the test loading, EL-Centro wave (E wave) and Wenchuan wave ( $\mathrm{W}$ wave) are used as the original seismic waves. The reciprocal power spectrum model is applied and the coherence between two points is used, and corrections are made in the original seismic waves based on the coherent amplitude and coherent phase angle to obtain the multipoint seismic wave time course at two different locations considering spatial correlation. Based on response spectra, then we produce its power spectra and Fourier amplitude spectra, and a nonstationary spatially artificial random multipoint seismic wave (R-wave) is obtained by iteration based on the Fourier transform and the intensity envelope function.

The two types of multipoint seismic waves were synthesized by using MATLAB, with a holding time of $1 / 5$ and a frequency and acceleration peak of 5 times the similarity ratio converted and adjusted. Input seismic waves were with acceleration peaks of $0.25 \mathrm{~g}, 0.50 \mathrm{~g}, 1.00 \mathrm{~g}$, and $1.55 \mathrm{~g}$ to simulate $7^{\circ}, 8^{\circ}, 9^{\circ}$ and nine degrees rare earthquakes, respectively. The test was divided into two cases of homogeneous and in-homogeneous soils with transverse and lateral loading to analyze the characteristics and patterns of the uniform and multipoint seismic response of the pipe-soil model under different loading conditions. The data collected during the tests consisted mainly of the acceleration response of the pipe and soil, strain response of the pipe, displacement response of the soil, and soil pressure response of the pipe and soil contact surfaces. 


\section{Synthesis of Multipoint Seismic Waves Based on Specific Seismic Records}

$\mathrm{Qu}$ and Wang [8] modified the spatially correlated ground shaking synthesis method derived by Hao, proposing that it is necessary to considering the spatial correlation with the ground shaking of the other $n-1$ points when the time curve of $n$ points is to be generated, and Zhou et al. [9] further proposed that the seismic waves collected at each point on the ground during a single earthquake are coherent, but the phase angles of the seismic waves at each of their points are the same, all being the phase angles of the original seismic waves. Based on the above ideas, the modified spatially correlated multipoint seismic wave synthesis equation is

$$
\begin{aligned}
u_{j}(t) & =\sum_{m=1}^{n} \sum_{k=0}^{N-1} a_{j m}\left(\omega_{k}\right) \cos \left[\omega_{k} t+\theta_{j m}\left(\omega_{k}\right)+\phi_{k}\right], \\
j & =1,2, \ldots, n,
\end{aligned}
$$

TABle 1: Values of parameters of Du and Chen models.

\begin{tabular}{lcccc}
\hline Parameter & $T_{\mathrm{s}}$ & $\omega_{0}$ & $\omega_{g}$ & $\xi_{g}$ \\
\hline Value & $40(100)$ & 1.8 & 31.42 & 0.72 \\
\hline
\end{tabular}

where $\mathrm{a}_{j m}\left(\omega_{k}\right)$ is to consider the amplitude of the $k$ frequency classification related to the $j$ point and the $m$ point. $\theta_{j m}\left(\omega_{k}\right)$ is to consider the phase angle of the $k$ frequency component related to the $j$ point and the $m$ point. $\varphi_{k}$ is to consider the phase angle of the original ground motion.

$\mathrm{a}_{j m}\left(\omega_{k}\right)$ and $\theta_{j m}\left(\omega_{k}\right)$ can be obtained from the power spectrum matrix, and the power spectrum model is selected as the $\mathrm{Du}$ and Chen model [10] is from

$$
S(\omega)=\frac{1}{1+\left(T_{s} \omega\right)^{2}} \frac{1}{1+\left(\omega_{0} / \omega\right)^{2}} \frac{1+4 \xi_{g}^{2}\left(\omega^{2} / \omega_{g}^{2}\right)}{\left(1-\left(\omega^{2} / \omega_{g}^{2}\right)\right)^{2}+4 \xi_{g}\left(\omega^{2} / \omega_{g}^{2}\right)} S_{0}(M, R)
$$

where $T_{\mathrm{s}}$ is the time of ground motion; $\omega_{o}$ is the low frequency corner frequency; $\omega_{g}$ is the predominant period of the site; and $\varsigma_{g}$ is the damping ratio of the site. According to the type II site near earthquake, the values of each parameter are given in Table 1.

$S_{0}$ is the spectral intensity factor, which can be calculated from (3)-(6) based on its relationship with the mean maximum ground acceleration [11]:

$$
S_{0}=\frac{\bar{A}_{m}^{2}}{\beta \gamma^{2}}
$$

where

$$
\begin{aligned}
& \beta=\frac{4 \xi_{g}^{2} \omega_{h}+2 \xi_{g} \omega_{g}+\omega_{h}}{\omega_{h}^{2}+2 \xi_{g} \omega_{g} \omega_{h}+\omega_{g}^{2}} \frac{\pi \omega_{g} \omega_{h}}{2 \xi_{g}}, \\
& \gamma=\sqrt{2 \ln \left(\nu T_{s}\right)}+\frac{0.5772}{\sqrt{2 \ln \left(\nu T_{s}\right)}} \\
& v=\frac{1}{\pi} \sqrt{\frac{\omega_{h} \omega_{g}\left(8 \omega_{h} \xi_{g}^{3}+4 \xi_{g}^{2} \omega_{g}+\omega_{g}\right)}{4 \xi_{g}^{2} \omega_{h}+2 \xi_{g} \omega_{g}+\omega_{h}}} .
\end{aligned}
$$

The mean value of the maximum ground acceleration from (3) can be taken according to the correspondence relationship between seismic intensity and design basic seismic acceleration in the <Code for Seismic Design of Buildings > (GB 50011-2010) (2016 edition) according to the proposed input acceleration conditions on the shaking table from Table 2. $\omega_{h}$ is the spectral parameter that responds to the bedrock properties and takes the value of $25.13 \mathrm{rad} / \mathrm{s}$. The calculated power spectrum model is shown in Figure 2.

In addition to use existing power spectrum models, it is also possible to adopt the calculated power spectrum of a specific seismic record directly by the discrete Fourier transform function in the MATLAB, then multiplying the transform by its conjugate function to obtain the power spectrum of a specific seismic record. The EL-Centro and Wenchuan wave power spectra generated by the program are shown in Figures 3 and 4.

The reciprocal power spectrum of any two points $i, j$ in space is as follows:

$$
S_{i j}(i \omega)=\sqrt{S_{i}(i \omega) S_{j}(i \omega)}\left|\rho\left(\omega, d_{i j}\right)\right| e^{-i \omega\left(d_{i j} / v_{a}(\omega)\right)},
$$

where $S_{\mathrm{i}}(i \omega)$ and $S_{\mathrm{j}}(i \omega)$ are the self-power spectrum of points $i, j ; d_{i j}$ is the projection of the vector connecting the two points in the direction of seismic wave incidence; $v_{a}(\omega)$ is the apparent wave speed, a function of frequency, but generally taken as a fixed value for simplicity. In this paper, the apparent wave velocity equation using the $\mathrm{Qu}$ is calculated from (8), and the coefficients $c_{1}$ and $c_{2}$ take values of 3978 and 969 , respectively:

$$
v_{a}(\omega)=c_{1}+c_{2} \operatorname{In}\left(\frac{\omega}{2 \pi}\right)
$$

where $\rho\left(\omega, d_{i j}\right)$ is from (9), the hysteresis coherence function, and the coherence function model used in this paper is the Harichandran and Vanmareke model [12]: 
TABLE 2: Mean value of maximum ground acceleration $\left(\bar{A}_{m}\right)$.

\begin{tabular}{lcccc}
\hline Seismic fortification intensity & 6 & 7 & 8 & 9 \\
Mean value of maximum ground acceleration & $0.05(\mathrm{~g})$ & $0.10(\mathrm{~g})$ & $0.20(\mathrm{~g})$ & $0.40(\mathrm{~g})$ \\
\hline
\end{tabular}

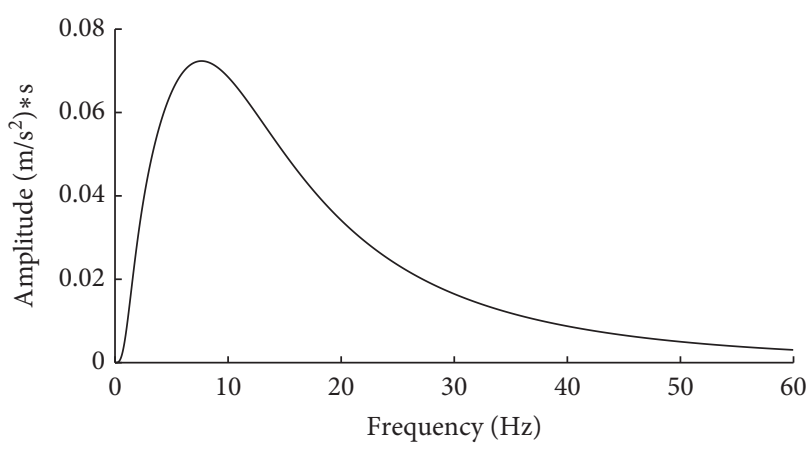

Figure 2: Power spectrum.

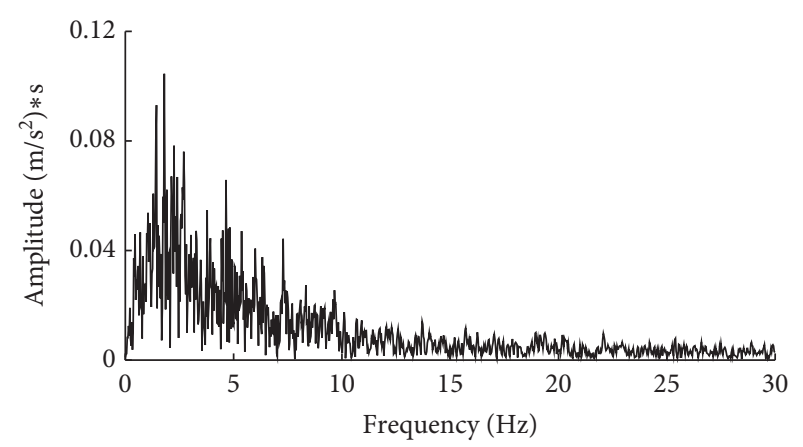

Figure 3: Power spectrum of EL-Centro wave.

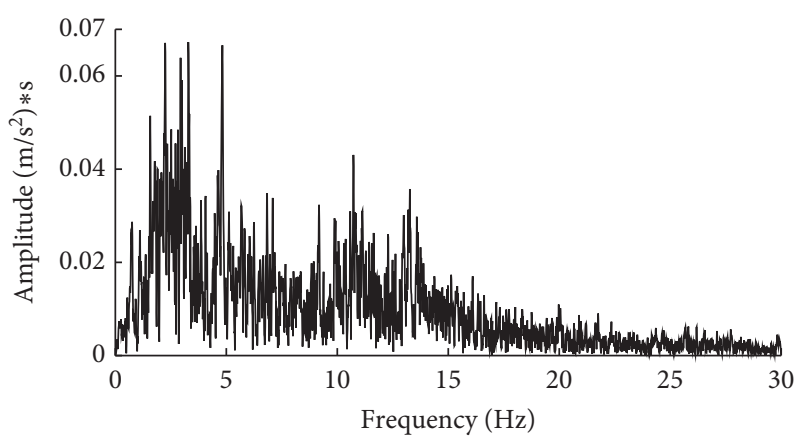

FIgURE 4: Power spectrum of Wenchuan wave.

$$
\begin{aligned}
\rho\left(\omega, d_{i j}\right)= & A \exp \left[-\frac{2 d_{i j}}{\alpha \theta(\omega)}(1-A+\alpha A)\right] \\
& +(1-A) \exp \left[-\frac{2 d_{i j}}{\alpha \theta(\omega)}(1-A+\alpha A)\right]
\end{aligned}
$$

where $A, \alpha, k$, and $b$ are regression coefficients. The statistics for Event 20 according to SMART-1 takes each parameter is given in Table 3.

From the above, the total power spectrum matrix considering the spatial characteristics of the two points can be obtained from (10), where the diagonal elements are obtained from the self-power spectrum model and the remaining elements are obtained from the mutual power spectrum model:

$$
S(i \omega)=\left[\begin{array}{cccc}
S_{11}\left(i \omega_{k}\right) & S_{12}\left(i \omega_{k}\right) & \ldots & S_{1 n}\left(i \omega_{k}\right) \\
S_{21}\left(i \omega_{k}\right) & S_{22}\left(i \omega_{k}\right) & \ldots & S_{21}\left(i \omega_{k}\right) \\
\vdots & \vdots & \vdots & \vdots \\
S_{n 1}\left(i \omega_{k}\right) & S_{n 2}\left(i \omega_{k}\right) & \ldots & S_{n n}\left(i \omega_{k}\right)
\end{array}\right] .
$$

By the property that $S(i \omega)$ is a Hermitian matrix and is positive definite, a Cholesky decomposition of it gives

$$
S(i \omega)=L(i \omega) L^{H}(i \omega)
$$

where

$$
L(i \omega)=\left[\begin{array}{cccc}
l_{11}\left(i \omega_{k}\right) & 0 & \ldots & 0 \\
l_{21}\left(i \omega_{k}\right) & l_{22}\left(i \omega_{k}\right) & \ldots & 0 \\
\vdots & \vdots & \vdots & \vdots \\
l_{n 1}\left(i \omega_{k}\right) & l_{n 2}\left(i \omega_{k}\right) & \cdots & l_{n n}\left(i \omega_{k}\right)
\end{array}\right] .
$$

Then, $\mathrm{A}$ and $\mathrm{B}$ can be determined according to the following equation [13]:

$$
\begin{aligned}
& a_{j m}\left(\omega_{k}\right)=\sqrt{4 \Delta \omega}\left|l_{j m}\left(i \omega_{k}\right)\right|, \\
& \theta_{j m}\left(\omega_{k}\right)=\arctan \frac{\operatorname{Im}\left[l_{j m}\left(i \omega_{k}\right)\right]}{\operatorname{Re}\left[l_{j m}\left(i \omega_{k}\right)\right]} .
\end{aligned}
$$

From (13) and (14) and the calculated phase spectrum $\left(\varphi_{k}\right)$ of the original ground shaking from (1), the time course of the ground shaking at point $j$ can be obtained. The phase spectra of EL-Centro and Wenchuan waves obtained by applying the angle() function in the MATLAB according to the relevant theory are shown in Figures 5 and 6 .

Using the above method, a multipoint ground vibration time history with a distance of $30 \mathrm{~m}$ was synthesized based on the two tables being $3 \mathrm{~m}$ apart. The acceleration time equations corresponding to the $7^{\circ}$ seismic intensity input to the two tables are then processed according to the similarity relationship and are from Figure 7.

\section{Synthesis of Artificial Random Multipoint Excitation Seismic Waves}

The synthesis of artificial random multipoint excitation seismic waves (R-wave) for testing can be started from the response spectrum. Firstly, the target response spectrum for synthesizing the ground shaking time history is determined with the peak acceleration time history corresponding to the seismic intensity as shown in Table 1 and other parameters such as site conditions, and based on the approximate conversion relationship between the response spectrum and the power spectrum, a mutual power spectrum matrix is 
TABLE 3: The values of each parameter of the coherence function model.

\begin{tabular}{lccccc}
\hline Parameter & $A$ & $\alpha$ & $k$ & $\omega_{0}$ & $b$ \\
\hline Value & 0.705 & 0.0263 & 257300 & 4.27 & 2.15 \\
\hline
\end{tabular}

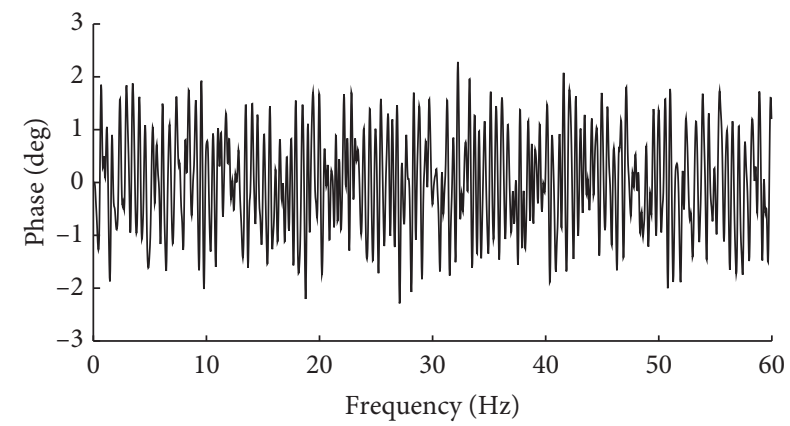

FIgURe 5: Phase spectrum of EL-Centro wave.

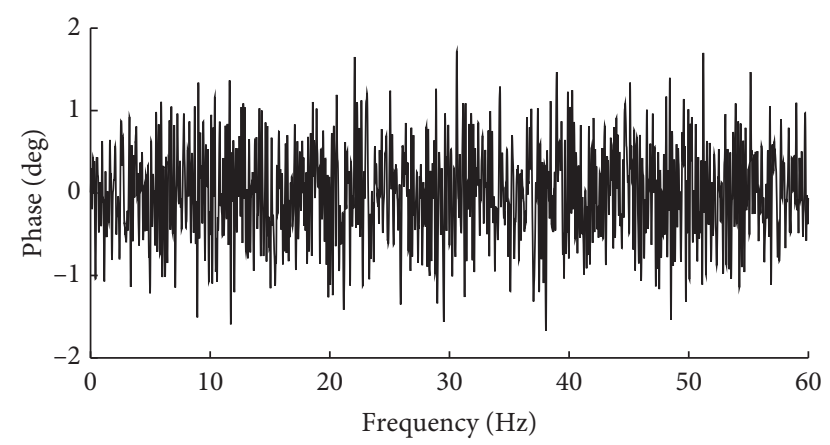

Figure 6: Phase spectrum of Wenchuan wave.

generated using the coherence function to form the total power spectrum matrix. The power spectrum matrix is then used to generate the Fourier amplitude spectrum at each point, and a fast Fourier transform is performed from the amplitude and phase spectra to obtain the smooth acceleration time history at each point. The smooth time course is calculated by multiplying the smooth time course by the intensity envelope function to obtain the ground shaking time course that satisfies the time-frequency nonsmoothness. Finally, the final acceleration time history is output by long-period filtering and baseline adjustment [14].

The reaction spectrum can be constructed by referring to the relevant formulate in the seismic codes of various industries such as construction, bridge, and water conservancy. In this paper, the reaction spectrum is calculated following the relevant contents of the <Guidelines for Seismic Design of Highway Bridges>(JTG/T B02-01-2019), given in the following equation:

$$
S= \begin{cases}S_{\max }(5.5 T+0.45), & T<0.1 s, \\ S_{\max }, & 0.1 s \leq T \leq T_{g}, \\ S_{\max }\left(T_{g} / T\right), & T>T_{g},\end{cases}
$$

where $S_{\max }$ is the maximum value of the acceleration response spectrum, $T$ is the self-oscillation period of the structure, and $T_{g}$ is the characteristic period. The calculation takes the site category as Class II and the characteristic period as $0.35 \mathrm{~s}$.

Referring to the specification, the one-sided power spectrum based on the design acceleration response spectrum can be estimated from

$$
S(\omega)=\frac{T \xi}{\pi^{2}} \frac{S^{2}}{\operatorname{In}\left[\left(-\left(T / 2 t_{d}\right) \ln p\right)^{-1}\right]},
$$

where $S$ is the design acceleration response spectrum; $P$ is the probability of not being exceeded and is taken as $0.5 ; t_{d}$ is the earthquake duration and $\zeta$ is the damping ratio; $t$ is the period; $\omega$ is the circular frequency. The power spectrum generated using the MATLAB programming is shown in Figure 8.

The mutual power spectrum, apparent wave velocity, coherence function, and total power spectrum matrices are constructed, and Cholesky decomposition is carried out from (7) to (13).

The phase spectrum required to synthesize seismic waves can be obtained by calculating the phase difference spectrum, which is related to the magnitude, epicenter distance, and site type. The relationship between the phase difference spectrum and the phase spectrum is as follows:

$$
\Delta \phi_{k}=\left\{\begin{array}{ll}
\phi_{k+1}-\phi_{k}, & 0 \leq \phi_{k+1}-\phi_{k}<2 \pi, \\
\phi_{k+1}-\phi_{k}+2 \pi, & -2 \pi \leq \phi_{k+1}-\phi_{k}<0,
\end{array} \quad(k=0,1, \ldots N-1),\right.
$$

where $N$ is the number of acceleration time points; $\Delta \phi_{k}$ is defined in the domain $[0,2 \pi]$.

In this paper, the phase difference spectrum model is using the Zhu-Feng model [15], which statistically analyses the mean numerical characteristics (mean deviation $\bar{\lambda}$ and standard deviation $\bar{\zeta}$ ) of the phase difference spectra of a large number of actual ground shaking records at different magnitudes and central distances, and determines that their phase difference spectra obey a log-normal distribution.

In this case, it is assumed that the phase difference spectra follow a normal distribution. In this case, it is assumed that $\Delta \phi_{k}=\ln \left(x_{k}\right)$, then the phase difference spectrum $x_{\mathrm{k}}$ follows a normal distribution, and its mean and standard deviation are calculated as follows: 


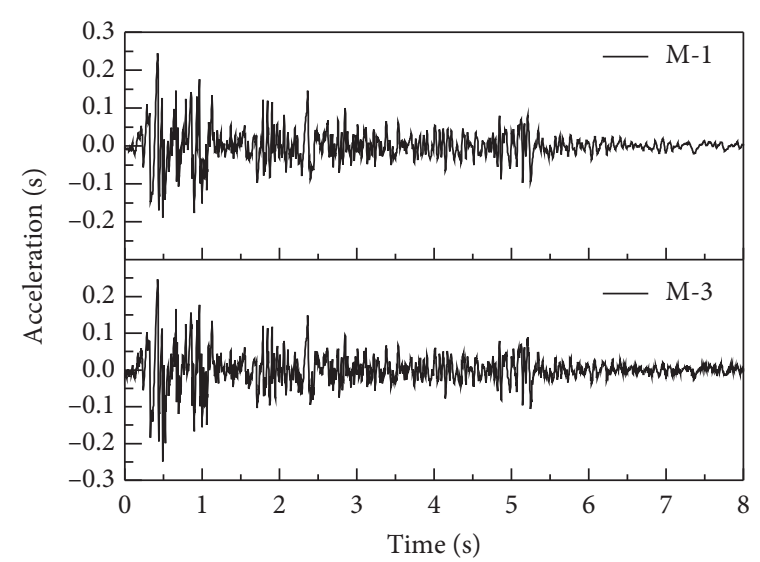

(a)

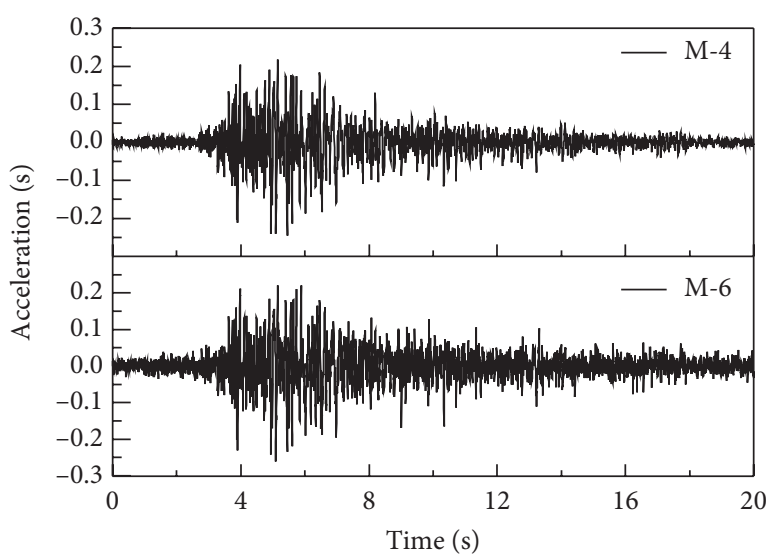

(b)

Figure 7: Multipoint excitation seismic wave synthesized based on specific seismic record. (a) EL-Centro wave; (b) Wenchuan wave.

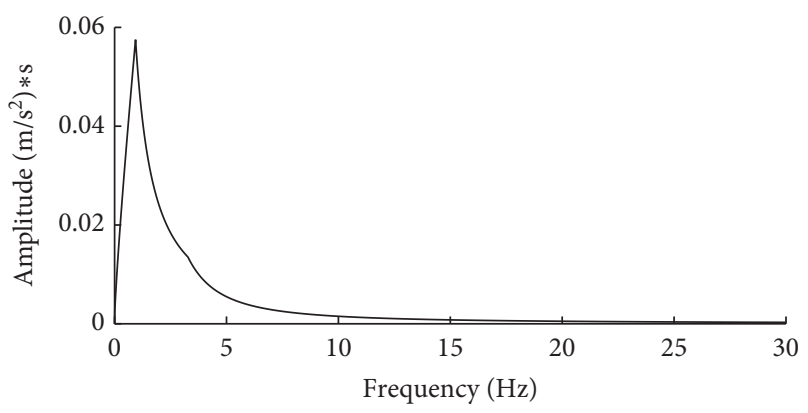

FIgURe 8: Power spectrum of artificial wave.

$$
\begin{aligned}
& \mu=\ln \bar{\lambda}-0.5 \ln \left(1+c^{2}\right), \\
& \sigma=\sqrt{\ln \left(1+c^{2}\right)} \\
& c=\frac{\bar{\zeta}}{\bar{\lambda}}
\end{aligned}
$$

In this equation, $c$ is the coefficient of variation, which reflects the dispersion of the random variable. The computer is first used to generate an overall distributed random number $\left(x_{\mathrm{k}}\right)$ that satisfies the above statistical characteristics and then invert $\left(\Delta \varphi_{\mathrm{k}}\right)$, and next, assuming that the initial phase angle is $\left(\varphi_{\mathrm{k}}=0\right)$, a series of phase angles can be derived using equation (18). The statistical parameters according to the magnitude of 6.5 and the epicenter distance of $60000 \mathrm{~m}$ are shown in Table 4 . The phase spectrum generated by the program using the angle() function in MATLAB is shown in Figure 9.

The Fourier spectral matrix of the smoothly vibrating field can be generated from the total power and phase spectra obtained from the following equation:

$$
f_{m}\left(\omega_{j}\right)=\sum_{i=1}^{m} \sqrt{\Delta \omega} L_{m r}\left(\omega_{j}\right) e^{i \phi_{r}\left(\omega_{j}\right)},
$$

where $\Delta \omega$ is the frequency step; $L_{m r}\left(\omega_{j}\right)$ B is the lower triangular matrix of the total power spectrum after Cholesky decomposition; $e^{i \phi_{r}\left(\omega_{j}\right)}$ is the phase spectrum.

A fast Fourier inversion of the above Fourier spectral matrix from (22) gives the smooth acceleration time history:

$$
x(t)=\sum_{-(N / 2)}^{N /(2-1)} f_{m}\left(\omega_{i}\right) e^{i \omega_{j} t_{k}} .
$$

The synthesized seismic waves are then nonsmoothly multiplied by the intensity envelope function from (23) [16]. After iteration based on the target response spectrum, the desired artificial random multipoint excitation seismic wave is obtained from Figure 10:

$$
\varphi(t)= \begin{cases}\left(t / t_{1}\right)^{2}, & 0 \leq t \leq t_{1}, \\ 1, & t_{1} \leq t \leq t_{2} \\ e^{-\alpha\left(t-t_{2}\right)}, & t_{2} \leq t \leq t_{d}\end{cases}
$$


TABLE 4: Statistical parameters of the phase difference spectrum.

\begin{tabular}{lccccc}
\hline Parameter & $\bar{\lambda}$ & $\bar{\zeta}$ & $\mathrm{c}$ & $\mu$ & $\sigma$ \\
\hline Value & 2.079 & 1.942 & 0.93 & 0.418 & 0.729 \\
\hline
\end{tabular}

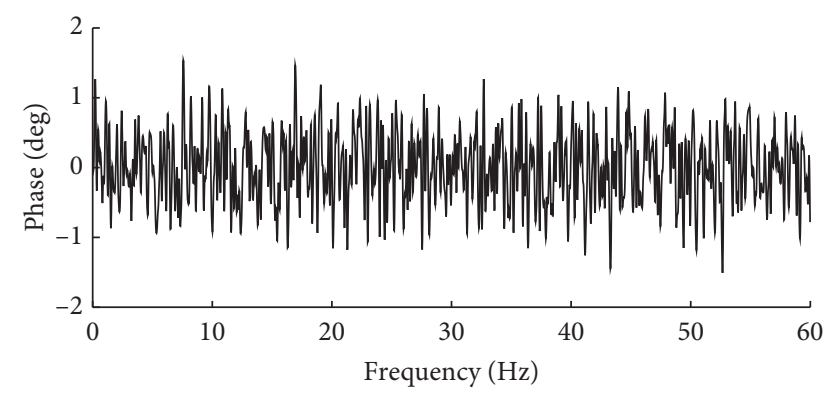

Figure 9: Phase spectrum of artificial multipoint seismic wave.

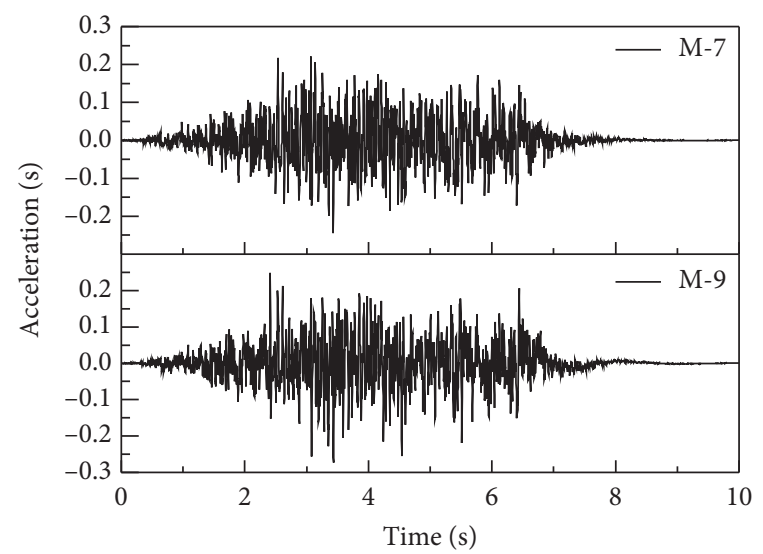

Figure 10: Synthetic artificial random multipoint excitation seismic wave.

where $t_{1}$ and $t_{2}$ are the peak duration time points, $t_{d}$ is the seismic wave duration, and $\alpha$ is the peak attenuation coefficient.

\section{Conclusion}

In this paper, a shaking table test for buried oil and gas pipelines under multipoint seismic excitation is developed, a shaking table loading scheme is formulated, and multipoint excitation seismic waves based on actual seismic records and artificial randomly simulated multipoint excitation seismic waves required for the test are synthesized; the main work and conclusions are as follows:

(1) A shaking table array test of seismic response of buried oil and gas pipelines under multipoint seismic excitation is designed, a multipoint excitation laminar shear continuum model soil box is developed, a multipoint excitation shaking table array seismic wave input scheme is formulated, and the test results show that the test scheme is reasonable and feasible.

(2) Based on the specific seismic records, the reciprocal power spectrum model is applied to make corrections in the original seismic waves based on the coherent amplitude and coherent phase angle, and the generated seismic wave time intervals considering spatial correlation at different locations can meet the requirements of the multipoint seismic excitation shaking table array test for buried oil and gas pipelines, and the test results have achieved the intended objectives.

(3) The experimental results show that the seismic wave time intervals obtained by using the coherence function based on the approximate conversion relationship between the response spectrum and the power spectrum can meet the requirements of the multipoint seismic excitation vibration array test for buried oil and gas pipelines.

\section{Data Availability}

The data used to support the findings of this study are available from the corresponding author upon request.

\section{Conflicts of Interest}

The authors declare that they have no conflicts of interest.

\section{Acknowledgments}

The authors gratefully acknowledge the National Natural Science Foundation of China (grant no. 51808446) (research on disaster mechanism and seismic performance of longdistance oil and gas pipelines under multidimensional and multisupport earthquake excitation) and Key Research and Development Program of Shaanxi (grant no. 2019JQ-055) (time-varying reliability analysis and optimal design of the oil and gas pipeline system in service based on stochastic process).

\section{References}

[1] J. Han, M. H. El Naggar, L. Li, B. Hou, J. Xu, and X. Du, "Design and commissioning of continuous soil box supported on shake tables array for testing long geostructures," Soil Dynamics and Earthquake Engineering, vol. 132, Article ID 106107, 2020.

[2] J. Han, M. H. El Naggar, B. Hou, X. Du, Y. Shuai, and L. Li, "Nonlinear soil response under non-uniform seismic excitation from multi-point shaking table tests," Soil Dynamics and Earthquake Engineering, vol. 139, Article ID 106342, 2020.

[3] Z. Aspasia, H. S. Alfredo, and Y. K. Wen, "Lifeline response to spatially variable ground motions," Earthquake Engineering \& Structural Dynamics, vol. 16, no. 3, pp. 361-379, 1988.

[4] I. Okawa, T. Kashima, H. Kitamura et al., "On the generation of the design earthquake ground motion time history," Engineering Geology, vol. 107, pp. 77-87, 2009.

[5] M. Hai, C. Jun, L. Jie et al., "Shaking table experimental study on non-uniform excitation of underground pipeline soil," Journal of underground space and engineering, vol. 5, pp. 852-859, 2008.

[6] N. J. Kerry, "Synthesis of seismic surface waves," Geophysical Journal International, vol. 64, no. 2, pp. 425-446, 1981. 
[7] G. Deodatis, "Proposed a simulation algorithm based on the spectral representation to generate sample functions that employ the mutual power spectral density function to generate non-smooth random seismic waves," Civil Engineering Journal, vol. 4, no. 1, pp. 239-257, 2017.

[8] T. Qu and Q. Wang, "Basic formula of spatial correlation multi-point ground motion synthesis," Earthquake Engineering and Engineering Vibration, vol. 1, pp. 8-15, 2012.

[9] R. Dong, J. Zhou, and X. Feng, "Study on synthesis method of non-stationary spatially correlated multi-point ground motions," Earthquake Engineering and Engineering Vibration, vol. 3, pp. 10-14, 2007.

[10] X. Du and H. Chen, "Stochastic simulation of ground motion and its parameter determination method," Earthquake Engineering and Engineering Vibration, vol. 4, pp. 1-5, 1994.

[11] J. Ou and H. Liu, "Random seismic response spectrum of structures based on random ground motion model and its application," Earthquake Engineering and Engineering Vibration, vol. 1, pp. 14-23, 1994.

[12] R. S. Harichanoran and E. H. Vanmarcke, "Stochastic variation of earthquake ground motion in space and time," Journal of Engineering Mechanics, vol. 112, no. 2, pp. 154-174, 1986.

[13] Y. Wu and Y. Gao, "Spatial correlation multi-point ground motion synthesis method based on power spectrum matrix square decomposition," Engineering Mechanics, vol. 29, no. 4, pp. 29-35, 2012.

[14] C. Fangliang, Seismic Vulnerability Analysis of High Pier Multi Tower Cable-Stayed Bridge Under Multi-Point Excitation, Hunan University, Changsha, China, 2018.

[15] Z. Yu and Q. Feng, "Digital characteristics of phase difference spectrum distribution of earthquake acceleration," Earthquake Engineering and Engineering Earthquake, vol. 2, pp. 30-37, 1993.

[16] Q. S. Yang and Y. J. Chen, "A practical coherency model for spatially varying ground motions," Structural Engineering \& Mechanics, vol. 9, no. 2, pp. 141-152, 2000. 